\title{
David trotzt Goliath
}

\section{Die kombinierte Erzeugung von Wärme und Strom in sogenannten Kraft-Wär- me-Kopplungsanlagen (KWK) stellt eine öko-effiziente Art der Energieerzeu- gung dar. Durch den Preisverfall seit der Liberalisierung des Strommarktes sind jedoch viele KWK-Anlagen bedroht. In jüngster Zeit erlassene gesetzliche Re- gelungen konnten den Abwärtstrend der KWK bislang nicht stoppen. Demge- genüber ist bei kleinen Blockheizkraftwerken laut einer aktuellen Marktanaly- se des IÖW bereits heute ein positiver Trend zu verzeichnen.}

$\mathrm{N}$ ropäischen Verband für Kraft-Wärme-Kopplung, sind in Deutschland seit der Liberalisierung des Strommarktes bereits 15 Prozent der KWKAnlagen stillgelegt oder gedrosselt worden, zudem sei die Zahl neuer KWK-Projekte um 25 bis 50 Prozent zurückgegangen. Dabei werden in Deutschland ohnehin nur etwa zehn Prozent des Stromes in KWK erzeugt; andere europäische Länder erreichen hier deutlich bessere Quoten (zum Beispiel Dänemark 50 Prozent, Niederlande 40 Prozent, Finnland 31 Prozent) (1).

Da mit KWK-Anlagen Wirkungsgrade von über 90 Prozent erreicht und dementsprechend Ressourcen gespart und Emissionen gesenkt werden können, setzt auch die Bundesregierung auf die KWK zur Erreichung des gesetzten Klimaschutzziels. Das Ökosteuergesetz sieht für KWK-Anlagen Steuererleichterungen bei der Strom-, Gas- und Mineralölsteuer vor. Dies reichte jedoch nicht aus, um die Konkurrenzfähigkeit gegenüber den großen Energieversorgern mit ihren abgeschriebenen Großkraftwerken und den geringen Stromerzeugungskosten zu erhalten. Um den dramatischen Rückgang der KWK zu verhindern, wurde im März das Gesetz zum Schutz der Stromerzeugung aus Kraft-Wärme-Kopplung (KWK-Gesetz) beschlossen.

Es verpflichtet Netzbetreiber, Strom aus KWK-Anlagen abzunehmen und mit 9 Pfennig pro Kilowattstunde zu vergüten. Der Abnahmepreis sinkt zu Beginn jedes neuen Jahres. Die Förderung ist an bestimmte Bedingungen geknüpft, die darauf hinaus laufen, dass nur ein geringer Teil aller KWK-Anlagen gefördert wird - und zwar im Wesentlichen diejenigen der ebenfalls durch die Liberalisierung stark unter Druck geratenen Stadtwerke. Daher wird das Gesetz in seiner jetzigen
Form von verschiedenen Verbänden scharf kritisiert. Aus ökologischer Sicht ist dabei zusätzlich zu bemängeln, dass sich die Förderung allein auf die Art der Stromerzeugung bezieht, unabhängig von der Effizienz der jeweiligen Anlage und unabhängig davon, ob die parallel erzeugte Wärme tatsächlich genutzt wird.

Das KWK-Gesetz stellt nur eine Übergangslösung dar; bis Ende des Jahres plant die Bundesregierung den Erlass eines KWK-Ausbaugesetzes, durch das der Bestand der KWK-Anlagen langfristig gesichert und ihr Anteil an der Stromerzeugung bis 2010 verdoppelt werden soll.

\section{- Kleine Anlagen im Aufwind}

Von der negativen Gesamttendenz im Bereich der KWK heben sich kleine Blockheizkraftwerke (BHKW) durch einen positiven Trend ab, wie die Befragung von 35 BHKW-Anbietern im Rahmen eines vom Umweltbundesamt geförderten IÖWProjektes (2) ergab. Blockheizkraftwerke sind Anlagen zur gekoppelten Erzeugung von Wärme und Strom, die Abwärme auf einem für die Gebäudeheizung geeignetem Temperaturniveau erzeugen und dezentral einsetzbar sind.

Für BHKW bis zu einer elektrischen Leistung von ca. 20 Kilowatt $(\mathrm{kW})$ überwiegen die Vorteile, die sich aus der Ökosteuer ergeben, die Nachteile aus der Strommarktliberalisierung. Zudem werden kleine BHKW - im Gegensatz zu großen KWK-Projekten - seltener durch Dumpingangebote von Seiten der Energieversorgungsunternehmen unwirtschaftlich gemacht.

Derzeit gibt es im Leistungsbereich bis $100 \mathrm{~kW}$ 5.000 bis 6.000 installierte BHKW, wovon etwa 4.000 auf den Bereich kleiner $10 \mathrm{~kW}$ entfallen. 1999 wurden in diesem Segment mindestens 1.500 Neuanlagen in Betrieb genommen. Marktführer in diesem Bereich ist die Firma SenerTec (Schweinfurt), die seit 1997 seriengefertigte
BHKW mit einer Leistung von 5 bis $5,5 \mathrm{~kW}$ anbietet, und seitdem jährlich mehr als 1000 Anlagen abgesetzt hat. Wesentlicher Grund für den Erfolg ist neben der Standardisierung des BHKW die Vermarktung über verschiedene Partner wie zum Beispiel Heizungsinstallateure.

Die BHKW im Leistungsbereich bis $100 \mathrm{~kW}$ werden zu ca. 60 Prozent mit Erdgas und zu ca. 40 Prozent mit Heizöl betrieben. Sonderbrennstoffe wie Flüssig-, Klär-, Deponie- oder Biogas, Pflanzenöl oder Planzenölmethylester nehmen eine Randstellung ein. Durch das Erneuerbare-Energien-Gesetz sind jedoch im Bereich der regenerativen Brennstoffe Zunahmen zu erwarten.

\section{Fazit}

Angesichts des drastischen Rückbaus im Bereich KWK ist dringender Handlungsbedarf geboten. Die Förderung sollte sich jedoch nicht an den Anbietern, sondern an der Effizienz der Anlagen orientieren. Die gegenläufigen Teilerfolge kleinerer BHKW lassen es ratsam erscheinen, das breite Spektrum der KWK im Hinblick auf geeignete Maßnahmen differenzierter zu betrachten sowie bereits greifende Erfolgsfaktoren zu berïcksichtigen und zu verstärken. Den kleinen BHKW sollte dabei auch deshalb eine bedeutende Rolle zugesprochen werden, weil durch die Dezentralisierung der Energieversorgung nicht nur ökologische Effizienzpotenziale erschlossen werden können, sondern auch eine Stärkung der Region und des regionalen Handwerks erreicht werden kann, die zum Ausbau oder zur Sicherung von Arbeitsplätzen beiträgt.

\section{Anmerkungen}

(1) Mez, Lutz/ Piening, Anette/ Traube, Klaus: Was kann Deutschland hinsichtlich eines forcierten Ausbaus der KraftWärme-Kopplung von anderen Ländern lernen, edition der Hans-Böckler-Stiffung 22, Düsseldorf 1999.

(2) Machbarkeitsstudie für neue Umweltzeichen für die Produktgruppe Blockheizkraftwerke, im Auftrag des Umweltbundesamtes, in Kooperation von IÖW und Hamburg Gas Consult, Projektlaufzeit bis November 2000.

\section{Die Autorlnnen}

Esther Hoffmann und Bernd Hirschl sind wissenschaftliche MitarbeiterInnen am Institut für ökologische Wirtschaftsforschung.

Kontakt: IÖW, Potsdamer Str. 105, 10785 Berlin. Tel. 030/ 884594-0, Fax 030/ 8825439, E-mail: Esther.Hoffmann@ioew.de, Bernd.Hirschl@heidelberg.ioew.de 
(c) 20I0 Authors; licensee IÖW and oekom verlag. This is an article distributed under the terms of the Creative Commons Attribution Non-Commercial No Derivates License (http://creativecommons.org/licenses/by-nc-nd/3.o/), which permits unrestricted use, distribution, and reproduction in any medium, provided the original work is properly cited. 